\title{
Conocimientos, creencias y prácticas sobre el flúor en padres y docentes de escolares
}

\author{
Knowledge, beliefs and practices about fluoride in parents and school teachers
}

\author{
Natalia Fortich-Mesa ${ }^{1}$, Vivi Hoyos-Hoyos ${ }^{2}$,Ángela Romero-Anaya ${ }^{2}$,Deicy Muñoz-Viloria ${ }^{2}$, Kenia Alarcón-Vitola ${ }^{2}$ ， \\ Luis Fernando Herrera-Vanegas ${ }^{3}$
}

Citación: Fortich-Mesa N, Hoyos-Hoyos V, Romero-Anaya A, Muñoz-Viloria D, Alarcón-Vitóla K, Herrera-Vanegas LF. Conocimientos, creencias y prácticas sobre el flúor en padres y docentes de escolares. Ustasalud 2020;19: 19-27.

Licencia Creative Commons

\section{(c) (1) (\$) $\Theta$}

\section{Resumen}

Determinar los conocimientos, creencias y prácticas sobre el flúor en padres de familia y docentes de escolares, a través de un estudio descriptivo de corte transversal, la muestra estuvo conformada por padres de familia y docentes de diversas escuelas de la ciudad de Cartagena, que aceptaron su ingreso por medio del consentimiento informado, se aplicó una encuesta adaptada por Mattos y colaboradores, incluía 42 ítems. Posteriormente, se procesaron los datos y estos fueron reportados en tablas de distribución de frecuencias y porcentajes.

Los conocimientos de flúor en ambos grupos de estudio estuvieron enfocados en su función y aplicación, así como del uso y beneficios de las cremas dentales con flúor. Sin embargo, presentaban desconocimiento acerca de suplementos con flúor, así como sobredosis de flúor y fluorosis dental en los padres de familia. En el ítem "la utilización de cremas dentales con flúor debe comenzar desde que el niño nace" fue reportado por los docentes en un $(35,7 \%)$, mientras que los padres de familia lo consideran cuando salen todos los dientes temporales $(29,9 \%)$. Por último, los participantes informan que utilizan crema dental Colgate ${ }^{\circledR}$, dos veces al día con una cantidad de crema correspondiente a toda la cabeza del cepillo dental. Se evidencian deficiencias en los conocimientos, creencias y prácticas en padres de familia y docentes de escolares, se hace necesario fortalecer y reorientar la educación en salud tanto a padres como a docentes, haciendo hincapié en los fluoruros tópicos como estrategia de prevención y tratamiento de la caries dental basada en el riesgo individual de cada paciente.

Palabras clave: Flúor, conocimientos, actitudes y práctica en salud, padres, docentes, promoción de la salud (DeCS).

\begin{abstract}
To determine the knowledge, beliefs and practices about fluoride in parents and teachers of schoolchildren, a cross-sectional study was conducted in parents and teachers of different schools in the city of Cartagena, who accepted their admission through informed consent, data were collected with a self-administered questionnaire, which consisted of twenty-four items, survey was applied by Mattos et al., the data were processed and these were reported in frequency and percentage distribution tables for the univariate analysis and Chi-square test for the bivariate analysis.

The knowledge about fluoride in both study groups was focused on its function and application, as well as it uses and benefits of fluoride toothpastes. However, the participants presented ignorance about fluoride supplements, as well as fluoride overdose and dental fluorosis in parents. In the item, "the use of fluoride toothpastes should begin since the child is born" was reported by teachers in (35.7\%) while parents consider it when all temporary teeth come out (29.9\%). Finally, participants report using Colgate ${ }^{\circledast}$ toothpaste, twice a day with an amount, corresponding to the entire toothbrush head, despite the diverse knowledge, attitudes and behaviors, that participants have, it is necessary to strengthen some aspects through educational interventions to improve new knowledge and practices in oral health.
\end{abstract}

Keywords: Fluorides, health knowledge, attitudes, practice, parents, faculty, health promotion (MeSH).
1 Odontóloga, magíster en Epidemiología Clínica. Corporación Universitaria Rafael Núñez, Cartagena (Colombia)

2 Odontóloga, Corporación Universitaria Rafael Núñez, Cartagena (Colombia)

3 Odontólogo, Corporación Universitaria Rafael Núñez, Cartagena (Colombia)

Autor de correspondencia:

Natalia Fortich Mesa

Correo electrónico:

natalia.fortich@curnvirtual.edu.co
Recibido para publicación: 1 de julio de 2020 Aceptado para publicación 23 de octubre de 2020 


\section{INTRODUCCIÓN}

La caries dental es la enfermedad crónica más común en la infancia y se caracteriza por el desequilibrio de la sustancia dental y el fluido de la placa circundante, lo que ocasiona una pérdida de mineral de la superficie dental, cuyo signo es la destrucción localizada de los tejidos duros, considerado así un problema de salud pública ${ }^{1-3}$. En Colombia se han reportado cifras variables, llegando a afectar más de tres cuartas partes de los niños y niñas evaluados, con diferentes niveles de severidad ${ }^{4}$. Este problema de salud bucal es motivo de preocupación, por cuanto se tiene evidencia de que compromete el bienestar, la calidad de vida y el adecuado crecimiento y desarrollo, por lo que continúa siendo uno de los problemas más importantes de salud pública ${ }^{5}$.

Los fluoruros son la forma iónica del elemento químico flúor. Actualmente, se considera que el principal mecanismo de acción del flúor es local, o sea, relacionado con su presencia y permanencia en la cavidad bucal, actuando en el proceso de desmineralización-remineralización de los dientes ${ }^{6,7}$. Existen estudios que muestran que la presencia constante del flúor en la saliva y en el fluido del biofilm dental, en su forma libre y soluble, reduce la cantidad de minerales perdidos durante la desmineralización y activa la relación dosis-respuesta durante la remineralización, por tanto, constituye una línea de defensa importante, seguido de la educación en salud oral ${ }^{8}$.

Diversos vehículos, como geles, barnices, espumas, soluciones de enjuague y pastas dentales han sido usados con la finalidad de proporcionar fluoruros para el control de la caries dental, desde intervenciones a nivel poblacional hasta medidas de cuidado oral en el hogar a nivel individual y medidas preventivas clínicas dirigidas ${ }^{9,10}$. Sin embargo, cuando se da una ingesta crónica durante los periodos de formación de los dientes puede desarrollarse desde puntos blancos o líneas mal definidas, hasta la presencia de opacidades de toda la superficie del diente que se pigmentan e incluso, conllevan pérdidas de fragmentos del esmalte, lo que puede producir toxicidad crónica conduciendo a una fluorosis dental ${ }^{11,12}$.

Por tanto, la promoción de la salud bucal debe ser prioridad para un buen cuidado en los niños, procurando educar a los padres, profesores, otros profesio- nales de la salud y todos aquellos que influencian a la población de mayor riesgo; promoviendo así el mantenimiento de hábitos saludables a través de estrategias educativas, logrando aumentar los conocimientos sobre salud e higiene dental para que transmitan lo aprendido al colectivo y la familia, con lo cual se convierten en verdaderos promotores de la salud, mediante la adquisición de información y desarrollo de habilidades, para estimular el cambio de comportamiento y actitud del individuo, desmitificando la creencia popular de la población ${ }^{13,14}$.

Investigaciones realizadas a nivel internacional soportan la falta de conocimientos en el tema, autores como Baltaci y colaboradores mostraron en su estudio que el $65,8 \%$ de los profesores estuvieron de acuerdo con que el fluoruro fortalece el esmalte dental ${ }^{15}$, mientras que autores como Liu y colaboradores evaluaron las prácticas de higiene oral, el uso actual y el conocimiento sobre la pasta dental con flúor entre escolares, padres y profesores, concluyendo que los padres y profesores que usaron pasta dental con flúor fue del $86,0 \%$ y $87,0 \%$, respectivamente, así como ellos confirmaron el efecto preventivo de la pasta dental con flúor ${ }^{16}$. Sin embargo, su falta de conocimiento parece ser un obstáculo importante para cumplir este papel. Por lo cual, el objetivo principal de este estudio fue determinar los conocimientos, creencias y prácticas sobre el flúor en padres de familia y docentes de escolares de diversas instituciones de la ciudad.

\section{MATERIALES Y MÉTODOS}

Se hizo un estudio observacional descriptivo de corte transversal. La población de estudio estuvo conformada por 164 padres de familia y 28 docentes de escolares matriculados en seis instituciones de la ciudad de Cartagena, durante el primer semestre del año 2017. Para determinar el número de padres de familia y docentes se realizó un muestreo no probabilístico por conveniencia. Se utilizaron como criterios de inclusión: padres de niños matriculados activos durante el procedimiento y docentes de primaria con vínculo laboral vigente y que aceptaran su participación voluntaria. Se excluyeron a padres con impedimento físico o mental que no pudieran diligenciar el documento, padres y docentes que proporcionarán datos incompletos o en su defecto se retiraron de algunas de las instituciones seleccionadas. 
Evaluación de los conocimientos, creencias y prácticas del flúor

Previo a la recolección de los datos, a los padres de familia y docentes se les explicó el objetivo de este, y cada uno de ellos aceptó su participación por medio del consentimiento informado. Se utilizó un instrumento validado a juicio de expertos y una prueba piloto por Liu y colaboradores ${ }^{15} \mathrm{y}$ adaptado por Mattos y colaboradores ${ }^{17}$, con un índice de confiabilidad de 0,99 , el cual consta de 24 ítems, los diez primeros midieron los conocimientos acerca del flúor, los cinco siguientes las creencias y los nueve últimos las prácticas del flúor. Se aplicó inicialmente a los profesores, auxiliares en las instalaciones de las instituciones educativas y posteriormente a los padres de familia que asistieron a las reuniones de cada uno de los escolares.

\section{Análisis estadístico}

Cada uno de los datos recolectados fue tabulado por medio del programa Microsoft Excel versión 2016 y a través del software SPSS V22, utilizando estadística descriptiva, teniendo en cuenta medidas de frecuencia y porcentaje para las variables de naturaleza cualitativa, y medidas de tendencia central acompañados de medidas de dispersión para las variables de cuantitativas.

\section{Consideraciones éticas}

La presente investigación toma en consideración la Declaración de Helsinki, con relación al respeto por la libertad del individuo en la participación del estudio y la Resolución 008430 de 1993 de la República de Colombia, en la cual representa una investigación sin riesgo donde se incluyeron técnicas y métodos de investigación documental retrospectivos de los individuos que participaron en el estudio. Así mismo, este estudio contó con la autorización y aval ético ante el Comité de Ética de la institución patrocinadora.

\section{RESULTADOS}

\section{Características sociodemográficas}

La población de estudio estuvo conformada por 192 participantes, de los cuales 164 eran padres de familia y 28 docentes pertenecientes a las instituciones educa- tivas, el 79,7\% de género femenino y 20,3\% masculino. Respecto a la edad, fue un promedio de 28,9 $\pm 8,2$ años con predominio del estrato socioeconómico 1 y $34,9 \%$ de nivel educativo, técnico (Tabla 1).

\section{Evaluación de los conocimientos, creencias y prácticas del flúor}

Al evaluar los conocimientos se observó que padres y docentes conocían acerca del flúor, su función y aplicación, así como del uso y beneficios de las cremas dentales con flúor. Sin embargo, presentaban desconocimiento entorno a los diversos suplementos con flúor, como sal de mesa y alimentos. Con relación a las temáticas de sobredosis de flúor y fluorosis dental los padres familia, en especial, reportaron insuficiencias en un $46,3 \%$ y $43,9 \%$, respectivamente (Tabla 2 ).

Por su parte, en relación con las creencias, el $64,6 \%$ afirmó que el flúor se puede obtener de los enjuagues bucales. Así mismo, los profesores consideran que la utilización de cremas dentales con flúor debe comenzar desde que el niño nace, mientras que los padres de familia afirman que cuando salen todos los dientes temporales (leche) en un 35,7\% y $29,9 \%$ respectivamente. Por último, los participantes reportan utilizar crema dental Colgate ${ }^{\oplus}, 2$ veces al día con una cantidad de crema correspondiente a toda la cabeza del cepillo dental. Sin embargo, el 54,9\% los padres de familia no recuerdan hace cuánto sus hijos recibieron la aplicación de flúor, por el contrario, el $57,1 \%$ de los docentes afirman que fue hace $<$ de 6 meses, a través de cubetas (geles) y por parte del odontólogo (Tabla 3).

\section{DISCUSIÓN}

El presente estudio permitió describir las características sociodemográficas y los conocimientos, creencias y prácticas sobre el flúor en padres de familia y profesores de escolares de diversas instituciones de la ciudad. Dado que el uso de fluoruros es uno de los métodos más comunes para la prevención de la caries dental mediante la utilización de cremas dentales, enjuagues y otros suplementos con alta concentración de flúor ${ }^{18,19}$, se ha evidenciado que una alta ingesta de esta en periodos prolongados puede provocar toxicidad y el desarrollo de fluorosis dental, lo cual se refleja en datos de prevalencia en 
Colombia que alcanzan el $62,2 \%$ a los 12 años y a los 15 años del 56,1\%, según el IV Estudio Nacional de Salud Bucal (ENSAB IV) ${ }^{20}$. Por lo cual se hace necesario conocer la información que manejan los padres y docentes para el cuidado de la salud bucal en los escolares.

Tabla 1. Características sociodemográficas de la población de estudio

\begin{tabular}{|c|c|c|c|c|c|c|}
\hline \multirow[t]{2}{*}{ Variable } & \multicolumn{2}{|c|}{ Global } & \multicolumn{2}{|c|}{ Padres } & \multicolumn{2}{|c|}{ Docentes } \\
\hline & $\mathbf{n}$ & $\%$ & $\mathbf{n}$ & $\%$ & $\mathbf{n}$ & $\%$ \\
\hline \multicolumn{7}{|l|}{ Grupo } \\
\hline Padre & 29 & 15,1 & 29 & 17,7 & 0 & 0,0 \\
\hline Madre & 124 & 64,6 & 124 & 75,6 & 0 & 0,0 \\
\hline Otro familiar & 11 & 5,7 & 11 & 6,7 & 0 & 0,0 \\
\hline Docente & 28 & 14,6 & 0 & 0 & 28 & 100 \\
\hline \multicolumn{7}{|l|}{ Sexo } \\
\hline Femenino & 153 & 79,7 & 134 & 81,7 & 19 & 67,9 \\
\hline Masculino & 39 & 20,3 & 30 & 18,3 & 9 & 32,1 \\
\hline \multicolumn{7}{|l|}{ Estrato socioeconómico } \\
\hline 1 & 152 & 79,2 & 146 & 89 & 6 & 21,4 \\
\hline 2 & 38 & 19,8 & 16 & 9,8 & 22 & 78,6 \\
\hline 3 & 2 & 1,0 & 2 & 1,2 & 0 & 0,0 \\
\hline 4 & 0 & 0,0 & 0 & 0 & 0 & 0,0 \\
\hline \multicolumn{7}{|l|}{ Zona de Influencia } \\
\hline 1 & 42 & 21,9 & 35 & 21,3 & 7 & 25,0 \\
\hline 2 & 3 & 1,6 & 3 & 1,8 & 0 & 0,0 \\
\hline 3 & 112 & 58,3 & 101 & 61,6 & 11 & 39,3 \\
\hline 4 & 12 & 6,3 & 9 & 5,5 & 3 & 10,7 \\
\hline 5 & 2 & 1,0 & 2 & 1,2 & 0 & 0,0 \\
\hline 6 & 21 & 10,9 & 14 & 8,5 & 7 & 25,0 \\
\hline \multicolumn{7}{|l|}{ Colegio } \\
\hline A & 45 & 23,5 & 38 & 23,1 & 7 & 25 \\
\hline B & 112 & 58,3 & 101 & 61,6 & 11 & 39,3 \\
\hline $\mathrm{C}$ & 11 & 5,7 & 8 & 4,9 & 3 & 10,7 \\
\hline $\mathrm{D}$ & 2 & 1,0 & 2 & 1,2 & 0 & 0,0 \\
\hline E & 22 & 11,5 & 15 & 9,1 & 7 & 25,0 \\
\hline \multicolumn{7}{|l|}{ Nivel académico } \\
\hline Primaria completa & 6 & 3,1 & 6 & 3,7 & 0 & 0,0 \\
\hline Bachiller & 18 & 9,4 & 18 & 11,0 & 0 & 0,0 \\
\hline Técnico & 67 & 34,9 & 67 & 40,9 & 0 & 0,0 \\
\hline Tecnólogo & 25 & 13,0 & 25 & 15,2 & 0 & 0,0 \\
\hline Pregrado & 30 & 15,6 & 26 & 15,9 & 4 & 14,3 \\
\hline Posgrado & 27 & 14,1 & 9 & 5,5 & 18 & 64,3 \\
\hline Especialista & 17 & 8,9 & 11 & 6,7 & 6 & 21,4 \\
\hline Magíster & 0 & 0,0 & 0 & 0,0 & 0 & 0,0 \\
\hline Doctorado & 2 & 1,0 & 2 & 1,2 & 0 & 0,0 \\
\hline
\end{tabular}




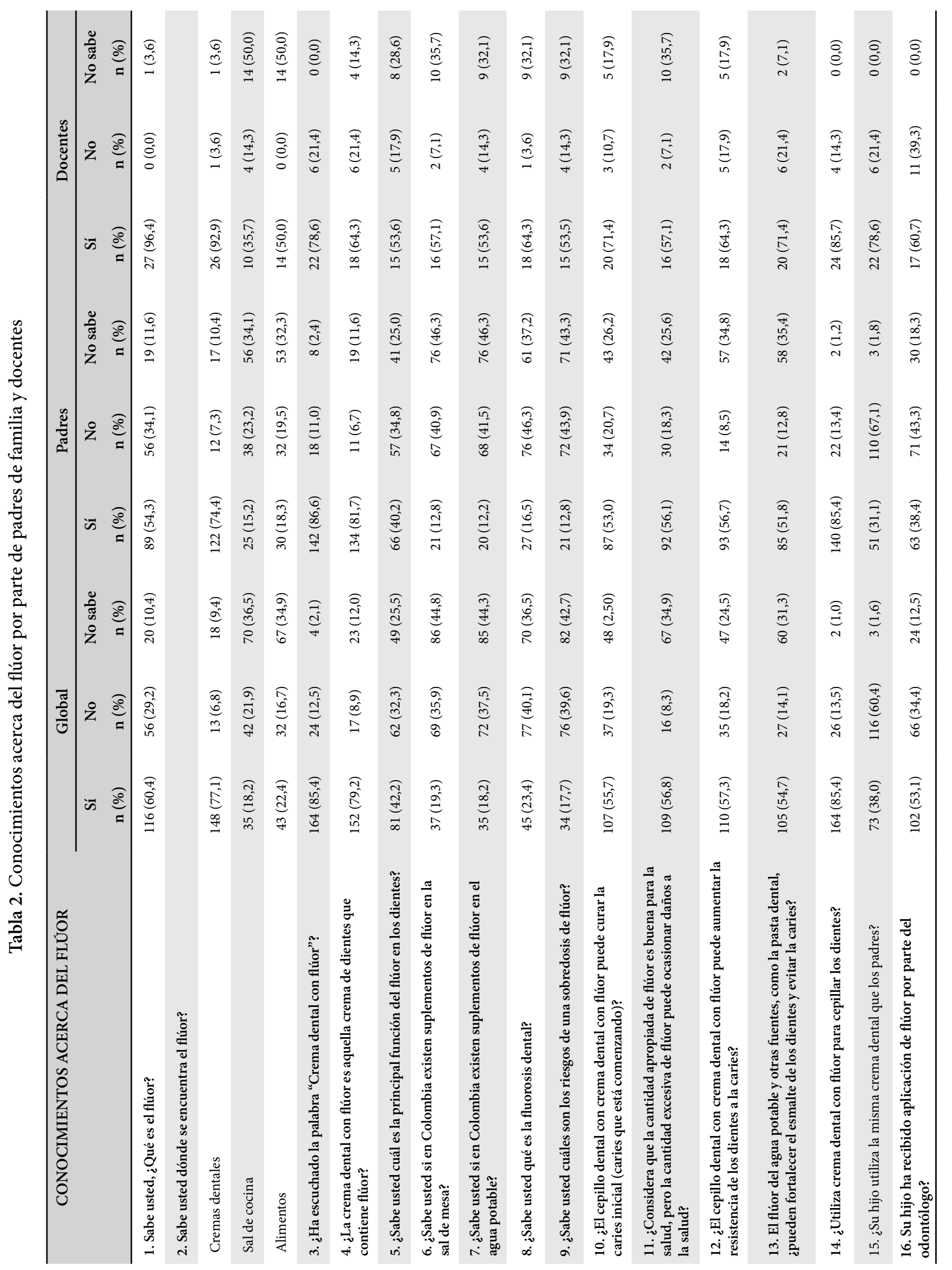


Tabla 3. Creencias y prácticas acerca de flúor por parte de padres de familia y docentes

\begin{tabular}{|c|c|c|c|}
\hline \multirow{2}{*}{ Ítems } & \multirow{2}{*}{ Global } & \multirow{2}{*}{$\begin{array}{c}\text { Padres } \\
\mathrm{n}(\%)\end{array}$} & \multirow{2}{*}{$\frac{\text { Docentes }}{\mathrm{n}(\%)}$} \\
\hline & & & \\
\hline \multicolumn{4}{|c|}{$\begin{array}{l}\text { 17. ¿De qué otra manera cree usted que se puede obtener el flúor además de la crema dental? (varias } \\
\text { opciones) }\end{array}$} \\
\hline Agua & $60(31,3)$ & $44(26,8)$ & $16(57,1)$ \\
\hline Sal de mesa & $58(30,2)$ & $41(25,0)$ & $17(60,7)$ \\
\hline Alimentos & $52(27,1)$ & $37(22,6)$ & $15(53,6)$ \\
\hline Enjuague bucal & $124(64,6)$ & $97(59,1)$ & $27(96,4)$ \\
\hline Otros & $28(14,6)$ & $16(9,8)$ & $12(42,9)$ \\
\hline \multicolumn{4}{|c|}{ 18. ¿Desde qué edad considera usted que es importante utilizar crema dental con flúor? } \\
\hline Desde que el niño nace & $15(7,8)$ & $5(3,0)$ & $10(35,7)$ \\
\hline Cuando sale el primer diente temporal (leche) & $48(25,0)$ & $44(26,8)$ & $4(14,3)$ \\
\hline Cuando salen todos los dientes temporales (leche) & $57(29,7)$ & $49(29,9)$ & $8(28,69$ \\
\hline Cuando sale el primer diente permanente (hueso) & $42(21,9)$ & $38(23,2)$ & $4(14,3)$ \\
\hline A cualquier edad es igual & $6(3,1)$ & $5(3,0)$ & $1(3,6)$ \\
\hline No sabe & $23(12,0)$ & $23(14,0)$ & $0(0,0)$ \\
\hline \multicolumn{4}{|l|}{ 19. ¿Qué marca de crema dental utiliza actualmente? } \\
\hline Colgate $^{\infty}$ & $74(38,5)$ & $65(39,6)$ & $9(32,1)$ \\
\hline Colgate $^{\infty}$ Luminous White & $4(2,1)$ & $4(2,4)$ & $0(0,0)$ \\
\hline Colgate $^{\circledast}$ Total 12 & $15(7,8)$ & $15(9,1)$ & $0(0,0)$ \\
\hline Colgate ${ }^{\star}$ Triple Acción & $63(32,8)$ & $53(32,3)$ & $10(35,7)$ \\
\hline $\mathrm{JGB}^{\circledR}$ Fluocardent & $16(8,3)$ & $14(8,5)$ & $2(7,1)$ \\
\hline Fortident Cuatri acción & $19(9,9)$ & $12(7,3)$ & $7(25,0)$ \\
\hline Crema dental para niños & $1(0,5)$ & $1(0,6)$ & $0(0,0)$ \\
\hline \multicolumn{4}{|l|}{ 20. ¿El día de ayer cuántas veces se cepilló usted los dientes? } \\
\hline Ninguna & $9(4,7)$ & $5(3,0)$ & $4(14,3)$ \\
\hline Una vez al día & $15(7,8)$ & $13(7,9)$ & $2(7,1)$ \\
\hline Dos veces al día & $110(57,3)$ & $93(56,7)$ & $17(60,7)$ \\
\hline$>$ de 2 veces en el día & $58(30,2)$ & $53(32,3)$ & $5(17,9)$ \\
\hline \multicolumn{4}{|l|}{ 21. ¿Qué cantidad de crema aplica? } \\
\hline Toda la cabeza del cepillo dental & $80(41,7)$ & $83(50,6)$ & $19(67,9)$ \\
\hline 1/2 de la cabeza del cepillo dental & $82(42,7)$ & $60(36,6)$ & $6(21,4)$ \\
\hline $1 / 4$ de cabeza del cepillo dental & $30(15,6)$ & $21(12,8)$ & $3(10,7)$ \\
\hline \multicolumn{4}{|l|}{ 22. ¿Hace cuánto su hijo recibió la aplicación de flúor? } \\
\hline$<$ de 6 meses & $45(23,4)$ & $29(17,7)$ & $16(57,1)$ \\
\hline$>$ de 6 meses & $18(9,4)$ & $18(11,0)$ & $0(0,0)$ \\
\hline$>$ de 12 meses & $33(17,2)$ & $27(16,5)$ & $6(21,4)$ \\
\hline No recuerda & $96(50,0)$ & $90(54,9)$ & $6(21,4)$ \\
\hline \multicolumn{4}{|l|}{ 23. ¿Qué tipo de flúor le aplicaron a su hijo? } \\
\hline Cubetas (gel de sabores) & $112(58,3)$ & $93(56,7)$ & $19(67,8)$ \\
\hline Barniz (pintura) & $80(41,79$ & $71(43,3)$ & $9(32,1)$ \\
\hline \multicolumn{4}{|l|}{ 24. ¿De dónde ha obtenido usted información acerca del flúor? } \\
\hline Odontólogo & $103(53,6)$ & $78(47,6)$ & $25(89,3)$ \\
\hline Medios de comunicación masivos & $27(14,1)$ & $24(14,6)$ & $3(10,7)$ \\
\hline Charlas educativas & $42(21,9)$ & $42(25,6)$ & $0(0,0)$ \\
\hline Amigos o familiares & $14(7,3)$ & $14(8,5)$ & $0(0,0)$ \\
\hline Droguerías & $6(3,1)$ & $6(3,7)$ & $0(0,0)$ \\
\hline
\end{tabular}


Mattos y colaboradores determinaron el nivel de conocimiento sobre pasta dental fluorada en padres y profesores de preescolares, reportando que el 91,0\% de la población total han escuchado la palabra pasta dental con flúor y sus beneficios con relación a la caries dental ${ }^{17}$. Igualmente, en el ítem cómo ha obtenido información sobre el flúor, se encontró que el odontólogo, seguido de los medios de comunicación masivos, fueron las fuentes más comunes tanto para los padres como para los docentes. Al correlacionar estos datos, se presentaron similitudes en torno a las ventajas del flúor en un $79,2 \%$, y la divulgación de este a través del odontólogo en un 53,6\%. Estos hallazgos pueden explicarse debido a que tanto padres como profesores buscan estar informados acerca de la higiene bucal de sus hijos, mediante las visitas y controles de odontología o por otros medios.

Otros autores como Baltaci y colaboradores evaluaron el conocimiento, las actitudes y los comportamientos de profesores de preescolares con respecto a la salud oral y dental, destacando así que el 50,4\% de los docentes consideran que la higiene dental en niños debe iniciar en la primera erupción dental, el $65,8 \%$ cree que el flúor fortalece el esmalte dental y así mismo, un $30,3 \%$ considera que el flúor puede tratar la caries inicial ${ }^{15}$. Por su parte, en el presente estudio, los profesores afirman que la higiene bucal debe realizarse desde que el niño nace en un $35,7 \%$ y el detenimiento de lesiones iniciales de caries en un $71,4 \%$.

En cuanto a los conocimientos de los padres de familia, consideran que la edad para utilizar crema dental con flúor es cuando salen todos los dientes temporales en un $29,9 \%$ y la cantidad de crema por aplicar es en toda la cabeza del cepillo dental en un 50,6\%, mientras que autores como Martínez y colaboradores destacan que el $33,3 \%$ de los padres iniciaron desde el nacimiento la higiene bucal en los niños y el $66,4 \%$ de los padres estuvieron en desacuerdo acerca de que la cantidad de crema dental apropiada para el cepillado del niño es menos de la mitad del largo del cepillo dental lo cual es similar con autores como Wiener y colaboradores ${ }^{21,22}$.

Por otro lado, Mota y colaboradores reportaron en su estudio que el 45,0\% de los docentes desconocen las pastas de dientes fluoradas, y los programas de fluoración del agua en las escuelas en un $78,9 \%{ }^{23}$. Por el contrario, el $64,3 \%$ de los docentes tienen conocimiento acerca de la crema dental con flúor y el 71,4\% utiliza la fluorización del agua para fortalecer el esmalte dental, lo que presenta concordancia con lo expuesto por Rodríguez y colaboradores, quienes concluyeron que los padres presentaron conocimientos acerca del flúor para prevenir y tratar la caries dental, así como el uso de crema dental y enjuagues con flúor, detener el progreso de la caries y aumentar su protección ${ }^{24}$. Además, Beljan y colaboradores afirman que el 71,7\% de los padres usaban crema de dientes con flúor y consideraron su uso dos veces al día de 1 a 3 minutos $^{25}$. Sin embargo, a pesar de los conocimientos que presentan ambos grupos de estudio, se recomienda mejorar las prácticas realizadas en los niños a través de intervenciones educativas con la finalidad de mejorar las falencias obtenidas.

Por último, es importante mencionar que una de las limitaciones más relevantes de esta investigación fue la dificultad en la recolección de la información hacia los docentes para participar en el estudio, lo cual repercutó en el tamaño de la muestra.

\section{CONCLUSIÓN}

De forma general se evidencian algunas deficiencias en los conocimientos, creencias, comprensión y prácticas en los padres de familia y docentes de escolares. Los conocimientos estuvieron asociados al flúor en términos de función y aplicación, así como del uso y beneficios de las cremas dentales con flúor. Sin embargo, se hace necesario mejorar algunos aspectos, como los efectos nocivos del flúor en altas concentraciones y los diversos suplementos disponibles en el mercado, debido a que los resultados encontrados fueron insuficientes, por lo cual es necesario reorientar los contenidos educativos y fomentar la educación en salud dirigida tanto a padres como a docentes, se debe hacer hincapié en los fluoruros tópicos a lo largo de todos los años, con especial atención en el uso tópico de flúor como estrategia de prevención y tratamiento de la caries dental, basada en el riesgo individual de cada paciente, así como el diseño de intervenciones educativas que permitan afianzar nuevos conocimientos y prácticas en la salud oral que logren ser eficaces a largo plazo y se vean reflejadas en los indicadores de salud oral de la población. 


\section{AGRADECIMIENTOS}

Los autores agradecen a los directores de las instituciones educativas y a los padres y docentes participantes en este estudio.

\section{REFERENCIAS}

1. Ramón RJ, Castañeda DM, Corona CMH, Estrada PGA, Quinzán LAM. Risk factors of dental decay in school children aged 5 to 11. MediSan. 2016;20(05):604-610.

2. Fejerskov O. Changing paradigms in concepts on dental caries: consequences for oral health care. Caries Res. 2004;38(3):182-91. DOI: 10.1159/000077753.

3. Kutsch VK. Dental caries: an updated medical model of risk assessment. J Prosthet Dent. 2014;111 (4):280-5. DOI: 10.1016/j.prosdent.2013.07.014.

4. Ramírez-Puerta BS, Escobar G, Franco AM, Ochoa EM, Otálvaro GJ, Agudelo AA. Caries dental en niños de 0-5 años del municipio de Andes, Colombia. Evaluación mediante el sistema internacional de detección y valoración de caries - ICDAS. Rev. Fac. Nac. Salud Pública. 2017;35(1):91-98. DOI: 10.17533/udea.rfnsp.v35n1a10.

5. Igoyen-Camacho ME, Luengas-Aguirre MI, Amador-Pedraza Y, Zepeda-Zepeda MA, Villanueva-Gutiérrez T, Sánchez-Pérez L. Comparación de barnices y dentífrico con flúor en la prevención de caries en escolares. Rev. Salud Pública. 2015;17(5):801-814. DOI: 10.15446/rsap. v17n5.4814.

6. Pollick H. The role of fluoride in the prevention of tooth decay. Pediatr Clin North Am. 2018;65(5):923-40. DOI: 10.1016/j.pcl.2018.05.014.

7. Pannuti CM, Cruz LR, Silva CGB, Romito GA, D'Hyppolito IM. Pasta dental con fluoruro de amina en la prevención de caries dental: revisión de la literatura. Braz J Periodontol. 2018;28(3):26-35.

8. Carey CM. Focus on fluorides: update on the use of fluoride for the prevention of dental caries. J Evid Based Dent Pract. 2014;14:95-102. DOI: 10.1016/j.jebdp.2014.02.004.

9. Whelton HP, Spencer AJ, Do LG, Rugg AJ. Fluoride revolution and dental caries: Evolution of policies for global use. J Dent Res. 2019;98(8):837-846. DOI: $10.1177 / 0022034519843495$.

10. Jones S, Burt BA, Petersen PE, Lennon MA. The effective use of fluorides in public health. Bull World Health Organ. 2005;83(9):670-6.

11. Moimaz AS, Saliba O, Marques LB, Garbin AS, Saliba A. Dental fluorosis and its influence on children's life. Braz. Oral Res. 2015;29(1):1-7. DOI:10.1590/18073107BOR-2015.vol29.0014.
12. Dobarganes AM, López N, Lima M, Calderón J, García ME. Fluorosis y caries dental en niños de 6 a 12 años. Revista Electrónica Dr. Zoilo E. Marinello Vidaurreta. 2015;40(3).

13. Cupé AC, García CR. Conocimientos de los padres sobre la salud bucal de niños preescolares: desarrollo y validación de un instrumento. Rev. Estomatol. Herediana. 2015;25(2):112-121.

14. Tolvanen M, Anttonen V, Matilla ML, Hausen H, Lahti S. Influence of children's oral health promotion on parents behaviours, attitudes and knowledge. Acta Odontol Scand. 2016;74(5):321 7. DOI: 10.3109/00016357.2015.1122836.

15. Baltaci E, Baygin O, Tuzuner T, Korkmaz FM. Evaluation of the knowledge, attitudes and behaviors of pre-school teachers on oral and dental health in the city center of Trabzon. Eur Oral Res. 2019;53(1):12-20. DOI: 10.26650/ eor.20199213.

16. Liu M, Zhu L, Zhang B, Petersen PE. Changing use and knowledge of fluoride toothpaste by schoolchildren, parents and schoolteachers in Beijing, China. Int Dent J. 2007;57(3):187-94. DOI: 10.1111/j.1875-595X.2007. tb00124.x.

17. Mattos M, Carrasco M, Valdivia G. Nivel de conocimiento sobre pasta dental fluorada en padres y profesores de preescolares. Int. J. Odontostomat. 2013;7(1):17-24. DOI: 10.4067/S0718-381X2013000100003.

18. González Martínez F, Arrieta Vergara KM, Fortich Mesa N. Factores familiares asociados con la prevalencia de Fluorosis dental en niños escolares en Cartagena-Colombia. Revista Clínica de Medicina de Familia. 2013;5(3):18290. DOI: 10.4321/S1699-695X2012000300006.

19. IV Estudio Nacional de Salud Bucal - ENSAB IV: Situación de salud bucal: para saber cómo estamos y saber qué hacemos. Bogotá: Ministerio de Salud y Protección Social de Colombia; 2013-2014.

20. Martínez F, Barrios CC, Salinas LE. Conocimientos, actitudes y prácticas en salud bucal de padres y cuidadores en hogares infantiles, Colombia. Salud Pública Méx. 2011;53(3):247-257.

21. Wiener RC, Crout RJ, Wiener MA. Toothpaste use by children, oral hygiene, and nutritional education: an assessment of parental performance. J Dent Hyg. 2009;83(3):141-5.

22. Mota A, Oswal KC, Sajnani DA. Sajnani AK. Oral health knowledge, attitude, and approaches of pre-primary and primary school teachers in Mumbai, India. Scientifica (Cairo). 2016;2016:5967427. DOI: 10.1155/2016/5967427.

23. Rodríguez JJ, Berbesí DJ. Conocimientos de higiene oral de acudientes y su relación con caries en menores de 5 años. Rev. Fac. Nac. Salud Pública. 2018;36(2):7-17. DOI:10.17533/udea.rfnsp.v36n2a03. 
24. Beljan M, Puharic Z, Zulec M, Boric D, Neumuller KR. Parent's and children's behavior and knowledge about oral health. Acta Med Croatica. 2016;70(3):165-71.
25. O’Mullane DM, Baez RJ, Jones S, Lennon MA, Petersen PE, Rugg AJ, et al. Fluoride and Oral Health. Community Dent Health. 2016;33(2):6.

\section{Correo electrónico de los autores}

Natalia Fortich-Mesa: natalia.fortich@curnvirtual.edu.co Vivi Hoyos-Hoyos: vhoyosh11@curnvirtual.edu.co

Angela Romero-Anaya: aromeroa11@curnvirtual.edu.co Deicy Muñoz-Viloria:dmunozv11@curnvirtual.edu.co

Kenia Alarcón-Vitola: kalarconv11@curnvirtual.edu.co Luis Herrera-Vanegas: lherrerav11@curnvirtual.edu.co 